\title{
Características M orfométricas de las Gallinas Criollas de Comunidades Rurales del Sur del Ecuador
}

\author{
Morphometric Characteristics of Indigenous Chicken in Rural Communities \\ OF SOUTHERn EcuAdor
Gustavo Villacís Rivas ${ }^{1}$, Galo Escudero Sánchez ${ }^{2}$, Fredy Cueva Castillo ${ }^{1,2}$, Agusto Luzuriaga Neira ${ }^{1}$

\section{Resumen}

El presente estudio tuvo como objetivo estudiar la variabilidad morfológica de gallinas criollas del sur de Ecuador. Se trabajó con 200 individuos adultos (31 machos y 169 hembras), que fueron caracterizados con la ayuda de 14 descriptores para caracterización morfométrica propuestos por la FAO. Dentro de las mediciones, se seleccionaron el peso corporal y el largo del tarso por tener directa relación con las características productivas de interés para el estudio. Se identificó la presencia de 10 biotipos de gallinas criollas con diferencias físicas visibles entre ellas. Mediante análisis estadístico multivariado se determinó que los 10 biotipos se agrupan en tres bloques en el caso de las hembras y en dos bloques en el caso de los machos.

Palabras clave: características morfométricas, gallinas criollas, peso corporal, tarsos

\section{Abstract}

The present study aimed to study the morphological variability of indigenous chicken in the south of Ecuador. A total of 200 adult individuals (31 males and 169 females) were characterized with the support of 14 descriptors for morphometric characterization established by FAO. On these measurements, body weight and length of the tarsus were selected due to their direct relationship with the productive characteristics of interest to the study. Ten biotypes of indigenous chicken were identified having evident physical differences among them. By using a multivariate statistical analysis, the biotypes were grouped in three clusters for females and in two clusters for males.

Key words: morphometric characteristics, indigenous chicken, body weight, tarsus

\footnotetext{
${ }^{1}$ Centro de Biotecnología, ${ }^{2}$ Carrera de Medicina Veterinaria, Universidad Nacional de Loja, Ecuador

${ }^{3}$ E-mail: chazo1970@hotmail.com
}

Recibido: 9 de octubre de 2014

Aceptado para publicación: 14 de diciembre de 2015 


\section{INTRODUCCIÓN}

La crianza de aves de traspatio es una actividad importante en las comunidades rurales de países en desarrollo. Dentro de las especies animales que se explotan bajo este sistema, las gallinas son las más importantes, ya que sus productos se destinan principalmente al autoconsumo. Por ejemplo, en Cuba, más del $90 \%$ de las familias rurales con animales de traspatio poseen gallinas (Pérez y Polanco, 2003).

La avicultura es una actividad de importancia, por constituirse en una fuente de alimento de las familias campesinas, por su aporte a la economía familiar y por ser un importante recurso zoogenético del país (Villacís, 2012). Dada la amplia variabilidad genética de la gallina criolla, se vienen desarrollando diversos estudios sobre este recurso zoogenético nativo, partiendo de una identificación y caracterización de las variedades criollas, para llegar a determinar el potencial genético asociado a producción y resistencia a enfermedades (Villacís, 2012). Por otro lado, el estudio de la gallina criolla contribuye a la conservación de los recursos genéticos avícolas, toda vez que la sustitución de razas autóctonas por razas comerciales constituye una amenaza real a los recursos genéticos avícolas mundiales (FAO, 2014).

El presente estudio tuvo por objetivo el estudio de la variabilidad morfológica de grupos poblacionales definidos de gallinas criollas del Sur de Ecuador.

\section{MATERIALES y Métodos}

El estudio fue realizado en seis comunidades rurales del sur del Ecuador: Bolaspamba, Cazaderos, Garza Real, Limones, Mangahurco y Paletillas, pertenecientes al cantón Zapotillo de la provincia de Loja. La zona posee un clima subtropical seco, con dos periodos climáticos definidos: seco y llu- vioso. Las unidades de producción campesinas seleccionadas para el estudio usualmente realizan un manejo tradicional para la crianza de las gallinas.

La zona en estudio se dividió en 83 cuadrantes de $5 \mathrm{~km}$ cada uno, donde se realizó un muestreo aleatorio de 380 predios campesinos, con la ayuda del software Win Episcope v. 2.0, durante el periodo comprendido entre abril y julio de 2013. Se georreferenció cada uno de los predios. Asimismo, solo se seleccionaron gallinas y gallos en edad reproductiva que pertenecían a uno de los biotipos en estudio, a través de un muestreo selectivo de individuos.

\section{Caracterización Morfométrica}

Del total de 14 descriptores morfométricos analizados en el estudio y que fueron propuestos por FAO (1981) y adaptados por Pérez y Polanco (2003) y Lázaro et al. (2012), se seleccionaron dos descriptores que tienen directa relación con la diferenciación morfológica de las gallinas, como son el peso corporal (g) y el largo del tarso $(\mathrm{cm})$.

Los descriptores morfométricos fueron el peso (g), largo corporal, perímetro pectoral, largo del muslo, largo de la pierna y del tarso, largo y ancho del ala, altura de la cresta, largo y ancho de la orejuela, largo y ancho de la barbilla, y largo de la cola; todos estos expresados en centímetros. Además, se analizaron las siguientes características fanerópticas: color y tipo de pluma, tipo de cresta y color de tarso, piel, pico y de los huevos.

Se trabajó con 169 hembras y 31 machos. Las aves fueron pesadas y medidas de manera individual, empleando una balanza digital con capacidad de $4000 \mathrm{~g}$ y $0.003 \mathrm{~g}$ de resolución, un escalímetro con $0.02 \mathrm{~mm}$ de precisión y una cinta métrica. Los datos obtenidos fueron sometidos a un análisis estadístico inferencial, utilizando análisis de varianza, prueba de Tukey y NPMANOVA en el programa SPSS v. 21 (IBM). En cada 
Cuadro 1. Medidas morfométricas (promedio \pm desviación estándar, en $\mathrm{cm}$ ) registradas en gallinas de biotipos criollos de comunidades rurales del sur del Ecuador

\begin{tabular}{|c|c|c|c|c|c|c|c|c|}
\hline Parámetro & $\begin{array}{c}\text { Guarica } \\
(n=28)\end{array}$ & $\begin{array}{l}\text { Enana } \\
(n=11)\end{array}$ & $\begin{array}{c}\text { Barbona } \\
\quad(n=8)\end{array}$ & $\begin{array}{l}\text { Copetona } \\
(\mathrm{n}=46)\end{array}$ & $\begin{array}{l}\text { Cubana } \\
\text { (n235) }\end{array}$ & $\begin{array}{c}\text { Fina } \\
(n=13)\end{array}$ & $\begin{array}{l}\text { Suta } \\
(n=9)\end{array}$ & $\begin{array}{l}\text { Calzada } \\
(n=23)\end{array}$ \\
\hline eso $(\mathrm{kg})$ & $1.84 \pm 0.34$ & $1.36 \pm 0.47$ & $2.37 \pm 0.33$ & $1.86 \pm 0.47$ & $2.27 \pm 0.77$ & $1.2 \pm 0.3$ & $1.65 \pm 0.38$ & $2.2 \pm 0.5$ \\
\hline $\begin{array}{l}\text { Largo } \\
\text { corporal }\end{array}$ & $44 \pm 9.6$ & $32.2 \pm 3.65$ & $43.0 \pm 2.8$ & $46.5 \pm 10.4$ & $42.9 \pm 8.4$ & $39.0 \pm 5.5$ & $37.2 \pm 4.0$ & $36.3 \pm 5.1$ \\
\hline $\begin{array}{l}\text { Perímetro } \\
\text { torácico }\end{array}$ & $31.9 \pm 2.2$ & $28.7 \pm 5.2$ & $34.0 \pm 1.4$ & $39.5 \pm 26.0$ & $35.6 \pm 4.5$ & $28.5 \pm 4.3$ & $32.5 \pm 6.2$ & $34.2 \pm 1.6$ \\
\hline $\begin{array}{l}\text { Largo de } \\
\text { muslo }\end{array}$ & $10.1 \pm 1.0$ & $9.2 \pm 1.3$ & $12.5 \pm 0.7$ & $10.4 \pm 1.3$ & $10.1 \pm 4.1$ & $9.6 \pm 0.5$ & $10.8 \pm 3.0$ & $13.3 \pm 2.3$ \\
\hline $\begin{array}{l}\text { Largo de } \\
\text { pierna }\end{array}$ & $11.7 \pm 1.5$ & $10.6 \pm 1.0$ & $13.5 \pm 0.7$ & $12.8 \pm 2.1$ & $13.8 \pm 2.9$ & $10.8 \pm 0.8$ & $11.8 \pm 1.5$ & $14.7 \pm 2.1$ \\
\hline $\begin{array}{l}\text { Largo de } \\
\text { tarso }\end{array}$ & $8.7 \pm 0.5$ & $6.2 \pm 0.8$ & $8.8 \pm 1.1$ & $9.9 \pm 5.8$ & $9.1 \pm 1.3$ & $7.4 \pm 0.4$ & $10.0 \pm 1.4$ & $9.0 \pm 0$ \\
\hline $\begin{array}{l}\text { Largo de } \\
\text { ala }\end{array}$ & $28.5 \pm 3.4$ & $27.6 \pm 4.4$ & $30.2 \pm 3.0$ & $31.3 \pm 5.0$ & $26.4 \pm 10.2$ & $29.7 \pm 3.6$ & $30.4 \pm 0.9$ & $29.0 \pm 5.0$ \\
\hline $\begin{array}{l}\text { Ancho de } \\
\text { ala }\end{array}$ & $21.2 \pm 1.2$ & $20.1 \pm 1.7$ & $21.8 \pm 1.1$ & $19.9 \pm 5.0$ & $20.6 \pm 5.3$ & $19.3 \pm 1.7$ & $18.9 \pm 1.7$ & $19.0 \pm 3.0$ \\
\hline $\begin{array}{l}\text { Altura de } \\
\text { cresta }\end{array}$ & $1.79 \pm 0.56$ & $1.6 \pm 1.32$ & $2.0 \pm 1.4$ & $2.07 \pm 1.12$ & $2.44 \pm 0.97$ & $1.6 \pm 0.46$ & $9.1 \pm 0.92$ & $2.5 \pm 0.5$ \\
\hline $\begin{array}{l}\text { Largo de } \\
\text { orejuela }\end{array}$ & $2.22 \pm 0.64$ & $1.8 \pm 0.61$ & $1.5 \pm 0$ & $2.33 \pm 0.96$ & $2.46 \pm 0.66$ & $10.4 \pm 0.27$ & $1.55 \pm 0.42$ & $1.66 \pm 0.57$ \\
\hline $\begin{array}{l}\text { Ancho de } \\
\text { orejuela }\end{array}$ & $1.54 \pm 0.33$ & $1.2 \pm 0.54$ & $1.62 \pm 0.35$ & $1.71 \pm 0.27$ & $1.7 \pm 0.4$ & $1.31 \pm 0.34$ & $1.37 \pm 0.29$ & $1.83 \pm 0.28$ \\
\hline $\begin{array}{l}\text { Largo de } \\
\text { barbilla }\end{array}$ & $1.57 \pm 0.37$ & $1.1 \pm 0.68$ & $1.1 \pm 1.3$ & $1.9 \pm 1.4$ & $2.08 \pm 0.84$ & $1.01 \pm 0.29$ & $1.32 \pm 0.19$ & $1.46 \pm 0.57$ \\
\hline $\begin{array}{l}\text { Ancho de } \\
\text { barbilla }\end{array}$ & $2.03 \pm 0.51$ & $2.06 \pm 0.83$ & $1.6 \pm 0.6$ & $2.13 \pm 0.78$ & $2.76 \pm 0.9$ & $1.41 \pm 0.21$ & $2.08 \pm 0.43$ & $2.03 \pm 0.64$ \\
\hline $\begin{array}{l}\text { Largo de } \\
\text { cola }\end{array}$ & $16.6 \pm 2.5$ & $16.9 \pm 2.1$ & $16.0 \pm 1.4$ & $17.1 \pm 2.7$ & $16.9 \pm 3.0$ & $19.1 \pm 1.4$ & 0 & $17.4 \pm 1.04$ \\
\hline
\end{tabular}

caso, se determinó el promedio y desviación estándar.

\section{Resultados y Discusión}

El uso de descriptores morfométricos permitió la identificación inicial de 10 biotipos de gallinas criollas, y que son conocidas por su denominación local como:

- Guarica o cuello desnudo: desprovistas de plumas en el cuello
- Shira o rizada: plumas dispuestas en sentido contrario

- Enana: con acortamiento de los huesos largos de sus piernas

- Barbona: con prolongación de las plumas a los lados del rostro y debajo del pico

- Calzada: plumas en tarsos y dedos

- Copetona: plumas largas en la cabeza a manera de copete

- Fina: de pequeño tamaño y destinada para el combate

- Suta: carente de rabadilla 
Cuadro 2. Medidas morfométricas registradas en gallos de biotipos criollos de comunidades rurales del sur del Ecuador

\begin{tabular}{lcccccc}
\hline Parámetro & $\begin{array}{c}\text { Guarica } \\
(\mathrm{n}=5)\end{array}$ & $\begin{array}{c}\text { Calzada } \\
(\mathrm{n}=7)\end{array}$ & $\begin{array}{c}\text { Barbona } \\
(\mathrm{n}=5)\end{array}$ & $\begin{array}{c}\text { Cubana } \\
(\mathrm{n}=9)\end{array}$ & $\begin{array}{c}\text { Fina } \\
(\mathrm{n}=4)\end{array}$ & $\begin{array}{c}\text { Suta } \\
(\mathrm{n}=6)\end{array}$ \\
\hline Peso (kg) & $2.6 \pm 0.5$ & $3.1 \pm 0.7$ & $2.4 \pm 1.3$ & $2.9 \pm 1.0$ & $1.5 \pm 0.2$ & $2.7 \pm 0.8$ \\
Largo corporal & $46.3 \pm 2.9$ & $49.0 \pm 2.8$ & $45.0 \pm 7.1$ & $65.5 \pm 4.5$ & $35.0 \pm 3.6$ & $49.5 \pm 6.6$ \\
Perímetro torácico & $38.1 \pm 3.4$ & $36.5 \pm 2.4$ & $44.0 \pm 8.5$ & $40.3 \pm 6.0$ & $25.5 \pm 3.5$ & $37.3 \pm 5.0$ \\
Largo de muslo & $11.5 \pm 1.3$ & $12.3 \pm 1.6$ & $12.0 \pm 4.2$ & $12.2 \pm 2.1$ & $10.5 \pm 1.7$ & $12.7 \pm 1.8$ \\
Largo de pierna & $14.5 \pm 1.9$ & $17.0 \pm 2.2$ & $16.5 \pm 4.9$ & $15.8 \pm 2.6$ & $11.1 \pm 1.0$ & $16.3 \pm 1.9$ \\
Largo de tarso & $9.5 \pm 1.3$ & $10.8 \pm 1.0$ & $10.5 \pm 2.1$ & $10.3 \pm 1.4$ & $8.4 \pm 0.5$ & $9.8 \pm 1.7$ \\
Largo de ala & $33.1 \pm 8.2$ & $36.9 \pm 6.8$ & $31.5 \pm 3.5$ & $35.0 \pm 5.5$ & $30.3 \pm 5.6$ & $39.0 \pm 2.9$ \\
Ancho de ala & $24.0 \pm 5.7$ & $24.5 \pm 0.7$ & $25.2 \pm 2.6$ & $22.7 \pm 2.9$ & $21.5 \pm 3.4$ & $25.1 \pm 1.6$ \\
Altura de cresta & $3.87 \pm 1.8$ & $4.26 \pm 2.36$ & $3.35 \pm 1.62$ & $2.77 \pm 2.58$ & $1.25 \pm 0.7$ & $5.23 \pm 1.56$ \\
Largo de orejuela & $2.12 \pm 1.83$ & $3.53 \pm 0.84$ & $2.5 \pm 0.7$ & $2.43 \pm 0.84$ & $1.55 \pm 0.52$ & $3.4 \pm 0.58$ \\
Ancho de orejuela & $1.75 \pm 0.72$ & $2.51 \pm 1.25$ & $2.0 \pm 0.7$ & $1.3 \pm 1.25$ & $1.35 \pm 0.28$ & $2.33 \pm 0.47$ \\
Largo de barbilla & $4.45 \pm 1.56$ & $3.71 \pm 1.61$ & $1 \pm 0$ & $3.66 \pm 2.48$ & $1.5 \pm 0.57$ & $4.23 \pm 1.36$ \\
Ancho de barbilla & $4.47 \pm 1.54$ & $4.16 \pm 1.36$ & $2 \pm 0$ & $4.51 \pm 1.9$ & $2.4 \pm 0.46$ & $4.05 \pm 0.47$ \\
Largo de cola & $24.9 \pm 3.1$ & $26.2 \pm 4.7$ & $24.0 \pm 9.8$ & $23.7 \pm 8.1$ & $30.3 \pm 14.8$ & 0 \\
\hline
\end{tabular}

Cuadro 3. Variables morfométricas en biotipos de gallinas criollas

\begin{tabular}{ccc}
\hline Grupo $^{1}$ & $\begin{array}{c}\text { Peso } \\
(\mathrm{g})\end{array}$ & $\begin{array}{c}\text { Largo de tarso } \\
(\mathrm{cm})\end{array}$ \\
\hline 1 & $3774^{\mathrm{a}}$ & $9.19^{\mathrm{a}}$ \\
2 & $1124^{\mathrm{c}}$ & $6.67^{\mathrm{c}}$ \\
3 & $1768^{\mathrm{b}}$ & $8.38^{\mathrm{b}}$ \\
\hline $\mathrm{a}, \mathrm{b}, \mathrm{c}$ & Letras dentro de columnas indican \\
& diferencia estadística $(\mathrm{p} \varangle 0.001)$ \\
1 & Por peso: 1: pesada; 2: liviana; 3: \\
semipesada. Por largo de tarso: 1: grande; \\
2: pequeña; 3: mediana
\end{tabular}

- Araucana o inauris: presenta un conjunto de plumas en la región auricular (poco frecuente en la zona de estudio)

- Cubana: no posee ninguna de las características antes descritas pero es similar a la gallina ibérica
Los biotipos encontrados en hembras se muestran en el Cuadro 1, con excepción de los biotipos shira y araucana, dado el reducido número de hembras encontradas. Asimismo, tampoco se incluyen las características fanerópticas que no fueron concluyentes para identificar los biotipos criollos del sur del Ecuador.

A diferencia de las hembras, se encontraron suficientes machos del biotipo calzada, pero no se hallaron machos de los biotipos copetón, enano, shiro y barbón (Cuadro 2). Asimismo, tampoco se muestran en el cuadro los descriptores fanerópticos que no resultaron definitivos para diferenciar los biotipos, pues estos son comunes para la mayoría de biotipos criollos.

En el análisis estadístico multivariado se determinó que las hembras de los biotipos criollos se agrupan en tres conglomerados (Fig. 1), considerando tanto los parámetros morfométricos de peso y largo de tarso (Cua- 


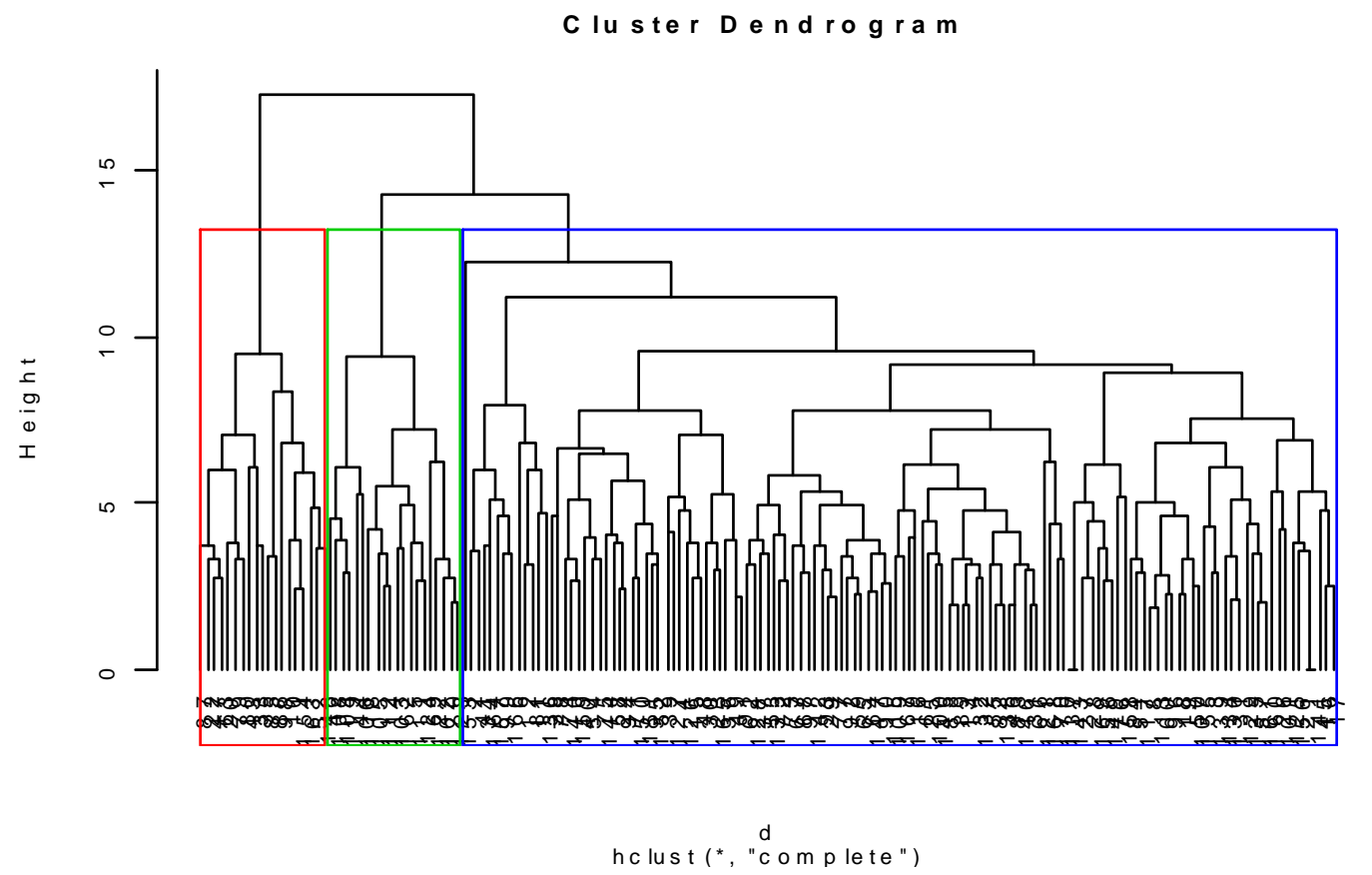

Figura 1. Conglomerados de gallinas criollas (hembras), según el análisis estadístico multivariado

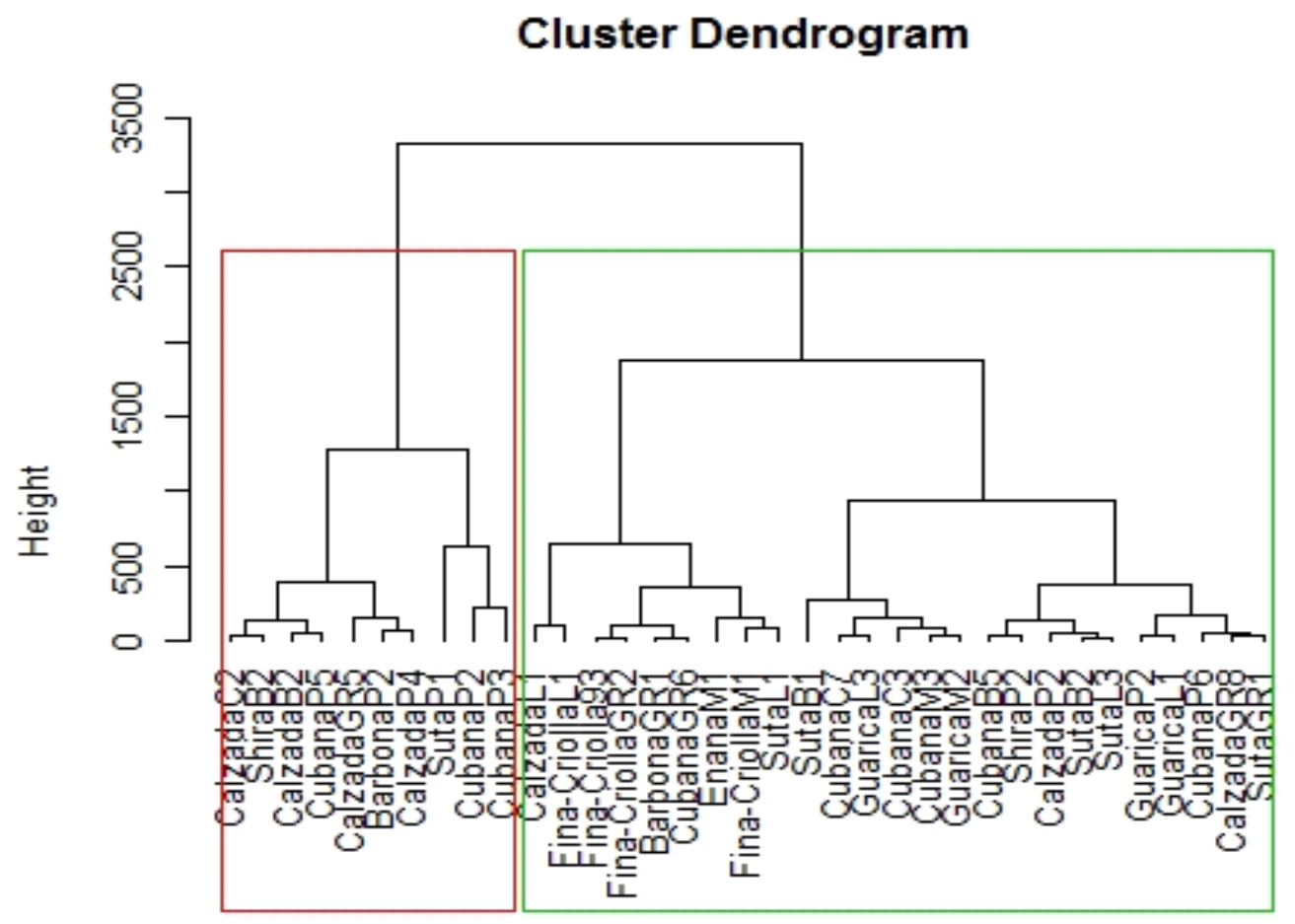

Figura 2. Conglomerados de gallos criollos (machos), según el análisis estadístico multivariado 
Cuadro 4. Variables morfométricas en biotipos de gallos criol los

\begin{tabular}{ccc}
\hline Grupo $^{1}$ & $\begin{array}{c}\text { Peso } \\
(\mathrm{g})\end{array}$ & $\begin{array}{c}\text { Largo de tarso } \\
(\mathrm{cm})\end{array}$ \\
\hline 1 & 3687.9 & 11.4 \\
2 & 2215.9 & 9.5 \\
\hline
\end{tabular}

${ }^{1}$ Por peso: 1: pesado; 2: liviano. Por largo de tarso: 1: grande; 2 : pequeño

dro 3). El análisis de varianza determinó que el Grupo 1 está constituido por hembras que en base a su peso pueden ser consideras como gallinas pesadas, el Grupo 2 como livianas y el Grupo 3 como semipesadas. En forma similar, para diferenciar los conglomerados de gallinas por tamaño de tarso, a estos grupos se les denominó gallinas grandes, pequeñas y medianas, respectivamente.

La prueba de análisis estadístico multivariado en la población de machos determinó la agrupación en dos conglomerados: el Grupo 1 de machos pesados y el Grupo 2 de machos livianos (Fig. 2; Cuadro 4). En forma similar, y basados en el tamaño del tarso de los machos, el análisis de varianza determinó la existencia de un grupo de machos grandes y otro de segundo grupo de machos pequeños (Cuadro 4).

Valencia (2011), en Colombia, identificó 13 sub-especies de gallinas criollas, basado en los trabajos de Dürigen (1971) y Hutt (1960), descartando las variedades dentro de una raza y proponiendo una reorganización para identificar los tipos de gallinas criollas. En el presente estudio, 10 de estas sub-especies fueron identificadas.

En varias poblaciones de gallinas de razas locales se reportan animales con cuello desnudo en frecuencias que van del 15 al $100 \%$ de la población (Pérez y Polanco, 2003), mientras que en Ecuador se señala una frecuencia de $14 \%$.
Zaragoza et al. (2013), en su caracterización zoométrica de gallinas locales de las tierras altas del sureste de México, identificaron varios de los biotipos presentes en Ecuador, como el biotipo normal o cubana, que al igual que en el presente estudio, resultó ser el de mayor frecuencia; además de los biotipos calzada, barbona y copetona. En el estudio mexicano, los pesos de los machos variaron entre 1260 y $4200 \mathrm{~g}$ y el de las hembras entre 1020 y $3050 \mathrm{~g}$, siendo de mayor y menor peso, respectivamente, que los valores encontrados en el presente estudio. Asimismo, Lázaro et al. (2012) reportan pesos inferiores a los del presente estudio en gallinas criollas del centro de México. Por otro lado, Pérez y Polanco (2003) encontraron una gran variabilidad de las características externas de la gallina indígena de Cuba, lo cual es frecuente en una población donde no se han realizado trabajos de selección y mejoramiento genético.

\section{Conclusiones}

- Se identificaron 10 biotipos de gallinas criollas en el sur del Ecuador.

- El manejo extensivo de las explotaciones avícolas tradicionales ha facilitado una alta endogamia entre las poblaciones de gallinas, de modo tal que un mismo individuo presenta características comunes de varios biotipos.

- Las características fanerópticas como color de pluma, color de tarsos, tipo de cresta y color de huevos no son definitivas para determinar el biotipo, dado que la mayoría de aves presenta características comunes entre los biotipos identificado en el presente trabajo.

- Los biotipos encontrados guardan similitud en peso y altura con gallinas criollas de Colombia, pudiendo sugerir un origen común. Por otro lado, las gallinas criollas de México son más pequeñas. 


\section{Literatura Citada}

1. Dürigen B. 1971. Tratado de avicultura. Especies y razas. Tomo I. Barcelona, España: Gustavo Gili. 731 p.

2. Hutt F. 1960. Genética avícola. Barcelona: Ed Salvat. 692 p.

3. Lázaro G, Hernández Z, Vargas L, Martínez L, Pérez A. 2012. Use of morphometric characters in the classification of local chickens. AICA 2: 109-114.

4. [FAO] Organización de las Naciones Unidas para la Agricultura y la Alimentación. 2014. Aves de corral y recursos zoogenéticos. [Internet]. Disponible en: http://www.fao.org/ag/againfo/ themes/es/poultry/AnGR.html

5. [FAO] Organización de las Naciones Unidas para la Agricultura y la Alimentación. 1987. Descriptores de especies avícolas. En: Banco de datos de recursos genéticos animales. Roma: FAO. 126 p.

6. Lázaro $G$, Hernández Z, Vargas L, Martínez L, Pérez A. 2012. Use of morphometric characters in the classification of local chickens. Actas Iberoamericanas de Conservación Animal AICA 2: 109-114.

7. Pérez A, Polanco G. 2003. La avicultura de traspatio en zonas campesinas de la provincia de Villa Clara, Cuba. Livest Res Rural Develop 15(2). [Internet]. Disponible en: http:// www.cipav.org.co//rrd/lrrd15/2/ pere152.htm

8. Valencia J. 2011. La gallina criolla colombiana. Palmira: Universidad Nacional de Colombia. 61 p. [Internet]. Disponible en: http://www.bdigital. unal.edu. co/3412/1/9789588095561.pdf

9. Villacís $\boldsymbol{G}$ 2012. La avicultura rural de la frontera sur ecuatoriana. Loja: Ed La Hora de Loja. [Internet]. Disponible en: http://dspace.unl.edu.ec/jspui/bitstream/ 123456789/21/3/gustavo\%20villacis\% 20rivas_2012.pdf

10. Zaragoza M, Rodríguez $H$, Hernández $Z$, Perezgrovas $G$, Martínez C, Méndez E. 2013. Caracterización de gallinas batsi alak en las tierras altas del sureste de México. Arch Zootec 62(239): 321-332. 